\title{
Resistência de Painéis Aglomerados de Acacia mangium Willd. Colados com Ureia-formaldeído e Taninos a Organismos Xilófagos
}

\author{
Fabricio Gomes Gonçalves ${ }^{1}$, Victor Fassina Brocco ${ }^{1}$, Juarez Benigno Paes ${ }^{1}$, \\ Pedro Licio Loiola ${ }^{2}$, Roberto Carlos Costa Lelis ${ }^{3}$ \\ ${ }^{1}$ Departamento de Ciências Florestais e da Madeira, Universidade Federal do Espírito Santo - UFES, \\ Jerônimo Monteiro/ES, Brasil \\ ${ }^{2}$ Programa de Pós-Graduação em Engenharia Florestal, Universidade Federal do Paraná - UFPR, Curitiba/PR, Brasil \\ ${ }^{3}$ Departamento de Produtos Florestais, Universidade Federal Rural do Rio de Janeiro - UFRRJ, Seropédica/RJ, Brasil
}

\begin{abstract}
RESUMO
Objetivou-se avaliar a resistência de aglomerados confeccionados com madeira de Acacia mangium Willd. colada com adesivo à base de ureia-formaldeído e taninos a organismos xilófagos. Os painéis foram produzidos com $40 \times 40 \times 1,27 \mathrm{~cm}$ (largura $\times$ comprimento $\times$ espessura); ao adesivo, foram adicionados 10,20 e $30 \%$ de taninos, sendo empregadas quatro razões de compactação $(1,39 ; 1,55 ; 1,73$ e 2,00$)$. Nos ensaios com fungos e térmitas, utilizaram-se amostras de $2,50 \times 2,50 \times 1,27 \mathrm{~cm}$ (largura $\times$ comprimento $\times$ espessura). Empregaram-se os fungos Gloeophyllum trabeum e Trametes versicolor (12 semanas de ensaio) e o térmita Nasutitermes sp. (45 dias de ensaio). Para o Gloeophyllum trabeum, os painéis com maior proporção de taninos e razão de compactação foram mais eficientes. Para o Trametes versicolor, não houve diferença entre os adesivos e razões de compactação utilizados. O Gloeophyllum causou maior perda de massa que o Trametes. Os térmitas consumiram completamente as amostras.
\end{abstract}

Palavras-chave: painéis reconstituídos, biodeterioração, fungos apodrecedores, térmita subterrâneo.

\section{Resistance of Acacia mangium Willd. Wood Particleboards Bonded with Urea-formaldehyde and Tannins to Wood-destroying Organisms}

\begin{abstract}
This study aimed to evaluate the resistance of particleboards made of Acacia mangium Willd. wood, bonded with urea-formaldehyde and tannins as adhesive, against xylophagous organisms. Panels of $40 \times 40 \times 1.27 \mathrm{~cm}$ (width $\mathrm{x}$ length $\mathrm{x}$ thickness) were produced; 10, 20 and $30 \%$ of tannins were added to the adhesive; four compaction ratios $(1.39 ; 1.55 ; 1.73$ and 2.00$)$ were employed. Samples of $2.50 \times 2.50 \times 1.27 \mathrm{~cm}$ (width $\mathrm{x}$ length $\mathrm{x}$ thickness) were used in the tests with fungi and termites. Gloeophyllum trabeum and Trametes versicolor (12 weeks) fungi and Nasutitermes sp. (45 days) termites were employed in the tests. Panels with the highest compaction ratio and proportion of tannins provided the most effective resistance to Gloeophyllum trabeum. No difference between the adhesives and compaction ratios was observed for Trametes versicolor. Gloeophyllum trabeum caused greater mass loss than Trametes versicolor. The samples were completely consumed by the termites.
\end{abstract}

Keywords: particleboard, biodeterioration, decay fungi, subterranean termite. 


\section{INTRODUÇÃO}

Os painéis de partículas de madeira surgiram na Alemanha, durante a Segunda Guerra Mundial, como forma de utilizar resíduos de madeira, por causa da dificuldade de obtenção de madeiras de boa qualidade para produção de madeira compensada (Iwakiri, 2005; Trianoski, 2010).

As vantagens deste tipo de painel é a eliminação dos efeitos da anisotropia da madeira e dos fatores redutores de resistência, como nós, madeira juvenil e inclinação de grã (Moslemi, 1974; Maloney, 1993; Trianoski, 2010), além da adequação das propriedades físico-mecânicas dos painéis pelo controle dos parâmetros de processo, como resina, geometria das partículas e grau de densificação (Dacosta, 2004).

A indústria de painéis de madeira é de grande importância para a economia brasileira, sobretudo pela dinâmica de novas tecnologias associada à geração de renda e emprego nos setores moveleiro e da construção civil (Vieira et al., 2012).

O crescimento da indústria moveleira, tanto nacional como internacional, impulsionou $\mathrm{o}$ surgimento das indústrias de chapas, nas quais se utilizam predominantemente madeiras de reflorestamentos, sendo as espécies dos gêneros Pinus e Eucalyptus as mais empregadas.

Para atender à demanda de matéria-prima, torna-se necessário não somente ampliar as áreas de plantio, mas também buscar outras espécies de rápido crescimento e a utilização de adesivos alternativos para a fabricação dos painéis (Lima et al., 2006; Ndazi et al., 2006; Pauleski et al., 2007; Melo, 2009).

Em painéis particulados, são empregados diferentes tipos de adesivos em função do destino final do produto, da qualidade desejável das chapas de partículas e dos custos de produção. Estes adesivos podem ser de origem animal, sintéticos termorrígidos e sintéticos termoplásticos. $\mathrm{Na}$ classe dos sintéticos termorrígidos, os adesivos à base de ureia-formaldeído, conforme mencionado por Tostes et al. (2004) e Melo et al., (2010), são empregados em $90 \%$ de todos os painéis aglomerados produzidos no mundo.

Em contrapartida, a crescente conscientização ambiental e a consequente busca por matérias renováveis vêm paulatinamente impulsionando também a utilização de adesivos à base de taninos vegetais, encontrados basicamente na madeira (cerne) e na casca de algumas espécies arbóreas. De acordo com Dunky \& Pizzi (2002), os adesivos de taninos já são utilizados industrialmente em alguns países, como: África do Sul, Austrália, Zimbábue, Chile, Argentina, Brasil e Nova Zelândia. Mesmo assim, uma das principais propriedades referentes à qualidade dos painéis à base de materiais lignocelulósicos é a sua susceptibilidade em serem atacados por organismos xilófagos (Melo et al., 2010).

Diante do exposto, objetivou-se avaliar a resistência de aglomerados confeccionados com madeira de acácia colada com adesivo à base de ureia-formaldeído e diferentes proporções de taninos a organismos xilófagos.

\section{MATERIAL E MÉTODOS}

\subsection{Obtenção e preparo do material}

Para a confecção dos painéis aglomerados, foi utilizada madeira de dez árvores de Acacia mangium Willd. proveniente de cultivo consorciado com $50 \%$ do híbrido Eucalyptus urophylla $\times$ Eucalyptus grandis, localizado no município de Santa BárbaraMG, coordenadas $43^{\circ} 21^{\prime} 19^{\prime \prime}$ W e $20^{\circ} 02^{\prime} 19^{\prime \prime} \mathrm{S}$, pertencente à Empresa Celulose Nipo Brasileira S.A., CENIBRA, estando o plantio com cerca de 50 meses de idade.

As árvores foram seccionadas em toretes e foram retiradas as cascas; os toretes foram transformados em discos de aproximadamente $5,0 \mathrm{~cm}$ de espessura e então fracionados em partículas. As partículas, após secas ao ar e atingir um teor de umidade de $\pm 15 \%$, foram quebradas em moinho de martelo com peneira de $9,35 \mathrm{~mm}$.

\subsection{Adesivos empregados e manufatura dos painéis}

Para a manufatura dos painéis, foram utilizadas as partículas que passaram pela peneira de $4,0 \mathrm{~mm}$ e que ficaram retidas na de $0,60 \mathrm{~mm}$. $\mathrm{O}$ material foi seco em estufa de circulação forçada a $65^{\circ} \mathrm{C}$ até 
teor de umidade pré-determinado de $5 \%$, sendo as partículas armazenadas em sacos plásticos hermeticamente fechados.

$\mathrm{O}$ adesivo utilizado foi a ureia-formaldeído (MDP1021, diluído a 10\%), com teor de sólidos de $64 \%$, $\mathrm{pH}$ de 8,5 , viscosidade de $371,86 \mathrm{cP}$ e densidade de $1,23 \mathrm{~g} . \mathrm{cm}^{-3}$. A proporção de adesivo utilizada na confecção dos painéis foi de 10\% em relação à massa seca das partículas. Empregou-se o sulfato de amônia $\left(\mathrm{NH}_{4}\right)_{2} \mathrm{SO}_{4}$ a $24 \%$, em proporção de $2 \%$ em relação à massa total de sólidos, como catalisador. O tanino utilizado foi em forma de pó, comercializado com o nome de Phenothan AP com 12\% de umidade, nas proporções de 10, 20 e 30\% para a massa de adesivo.

Os painéis foram produzidos com quatro razões de compactação. Os valores médios obtidos para a densidade aparente (umidade de 10\%) dos painéis produzidos foram $0,47,0,53,0,58$ e 0,68 g.cm , $^{-3}$ respectivamente, para cada razão de compactação $1,39,1,55,1,73$ e 2,00 . A razão de compactação foi obtida ao dividir a densidade do painel pela densidade das partículas que lhe deram origem $\left(0,34\right.$ g. $\left.\mathrm{cm}^{-3}\right)$, gerando painéis de quatro densidades diferentes. Na Tabela 1, constam as formulações dos adesivos e as razões de compactação utilizadas na confecção dos painéis.

Após a quantificação do material necessário (adesivo e partículas) a ser aplicado para cada tratamento, as partículas foram dispostas no interior de uma encoladeira. A aspersão do adesivo ocorreu por meio de uma pistola pneumática acoplada ao tambor. O tanino foi aplicado na forma de pó diretamente sobre as partículas nas quais a ureia-formaldeído já havia sido aplicada; foram definidos cinco giros do tambor para envolvimento do tanino à ureia, conforme descrito por Gonçalves (2012). As partículas encoladas foram pré-prensadas com auxílio de uma fôrma de madeira, com dimensões de $40 \times 40 \times 40 \mathrm{~cm}$, para a acomodação do colchão.

Os painéis foram produzidos no Laboratório de Painéis de Madeira da Universidade Federal Rural do Rio de Janeiro, com dimensões de $40 \times 40 \times 1,27 \mathrm{~cm}$ (comprimento $\times$ largura $\times$ espessura), em uma prensa hidráulica de pratos planos e horizontais com aquecimento elétrico. Os parâmetros de prensagem foram tempo de 8 minutos à temperatura de $140^{\circ} \mathrm{C}$ e pressão de 3,92 MPa. Para cada tratamento, foram produzidos quatro painéis.

Após a prensagem, os painéis foram mantidos em sala climatizada com umidade relativa de $65 \pm 5 \%$ e temperatura de $20 \pm 3^{\circ} \mathrm{C}$, até atingirem o equilíbrio. Retiraram-se aproximadamente $4 \mathrm{~cm}$ da borda das chapas, e os corpos de prova foram confeccionados visando à realização dos ensaios.

Tabela 1. Composição dos adesivos e a razão de compactação utilizadas na confecção dos painéis.

Table 1. Composition of adhesives and the compaction ratio employed on panel confection.

\begin{tabular}{|c|c|c|c|c|}
\hline \multirow{2}{*}{ Adesivo } & \multicolumn{2}{|c|}{ Composição (\%) } & \multirow{2}{*}{$\begin{array}{c}\text { Razão de } \\
\text { Compactação }\end{array}$} & \multirow{2}{*}{$\begin{array}{c}\text { Densidade Aparente } \\
\left({\left.\mathrm{g} . \mathrm{cm}^{-3}\right)}^{-3}\right.\end{array}$} \\
\hline & Ureia-Formaldeído & Taninos & & \\
\hline 1 & 100 & 0 & \multirow{4}{*}{1,39} & \multirow{4}{*}{0,47} \\
\hline 2 & 90 & 10 & & \\
\hline 3 & 80 & 20 & & \\
\hline 4 & 70 & 30 & & \\
\hline 1 & 100 & 0 & \multirow{4}{*}{1,55} & \multirow{4}{*}{0,53} \\
\hline 2 & 90 & 10 & & \\
\hline 3 & 80 & 20 & & \\
\hline 4 & 70 & 30 & & \\
\hline 1 & 100 & 0 & \multirow{4}{*}{1,73} & \multirow{4}{*}{0,58} \\
\hline 2 & 90 & 10 & & \\
\hline 3 & 80 & 20 & & \\
\hline 4 & 70 & 30 & & \\
\hline 1 & 100 & 0 & \multirow{4}{*}{2,00} & \multirow{4}{*}{0,68} \\
\hline 2 & 90 & 10 & & \\
\hline 3 & 80 & 20 & & \\
\hline 4 & 70 & 30 & & \\
\hline
\end{tabular}




\subsection{Ensaio de resistência a fungos apodrecedores}

O ensaio de resistência a fungos apodrecedores foi conduzido no Laboratório de Biodeterioração e Proteção da Madeira, localizado no Departamento de Ciências Florestais e da Madeira do Centro de Ciências Agrárias da Universidade Federal do Espírito Santo, em Jerônimo Monteiro-ES.

Para a realização do ensaio, foram selecionadas, aleatoriamente, seis amostras para cada combinação de adesivo e razão de compactação, com dimensões de $2,5 \times 2,5 \times 1,27 \mathrm{~cm}$ (comprimento $\times$ largura $\times$ espessura). As amostras foram secas em estufa a $50^{\circ} \mathrm{C}$ até atingirem massa constante.

O ensaio foi montado em frascos de $600 \mathrm{~mL}$, preenchidos com $300 \mathrm{~g}$ de solo de $\mathrm{pH} 5,7 \mathrm{e}$ capacidade de retenção de água de 33,89\%, conforme recomendado pela American Society for Testing and Materials - ASTM D - 1413 (2005a). O solo de cada frasco foi umedecido, conforme capacidade de retenção de água, sendo adicionados, por frasco, dois alimentadores de madeira de Pinus sp. Os frascos foram esterilizados a $121 \pm 2^{\circ} \mathrm{C}$, por 30 minutos.

Depois do resfriamento dos frascos, fragmentos obtidos de culturas puras dos fungos Gloeophyllum trabeum e Trametes versicolor foram inoculados nos alimentadores de madeira de Pinus sp. Após o desenvolvimento dos fungos nos alimentadores e a colonização no solo, foram adicionados os corpos de prova, à razão de duas amostras por frasco e seis repetições para cada combinação (tratamentos).

O ensaio foi mantido em sala climatizada $\left(28 \pm 2^{\circ} \mathrm{C}\right.$ e $75 \pm 5 \%$ de umidade relativa) por 12 semanas. Decorrido tal período, as amostras foram secas sob as mesmas condições anteriores à realização do ensaio. A perda de massa foi calculada e avaliada ao comparar os valores obtidos com os apresentados na Tabela 2 pela ASTM D - 2017 (2005b).

\subsection{Ensaio de preferência alimentar a térmitas xilófagos}

Para verificar a resistência dos painéis a térmitas xilófagos, foi montado o ensaio de preferência alimentar com o térmita subterrâneo Nasutitermes sp., conforme metodologia descrita por Paes et al. (2006, 2007).

Para a montagem do ensaio, os corpos de prova com dimensões de $2,5 \times 2,5 \times 1,27$ (comprimento $\times$ largura $\times$ espessura) foram distribuídos segundo um delineamento em blocos casualizados, em bandeja de polietileno disposta em uma caixa de cimento amianto de $250 \mathrm{~L}$ de capacidade, contendo uma camada de areia de aproximadamente $10 \mathrm{~cm}$ (Figura 1). As amostras ficaram expostas à ação dos térmitas durante 45 dias e, após o ensaio, foi avaliada a resistência dos painéis, conforme ASTM D - 3345 (2005c).

\subsection{Avaliação e análise dos resultados}

Para a avaliação da resistência dos painéis ao apodrecimento, foi utilizado um delineamento inteiramente casualizado com arranjo fatorial, em que foram avaliados os efeitos do tipo de adesivo, com quatro níveis; da razão de compactação, com quatro níveis, e do fungo utilizado, com dois níveis. Realizaram-se seis repetições para cada situação.

Para o ensaio com térmitas, foi empregado um delineamento em blocos casualizados com arranjo fatorial, em que foram avaliados os efeitos do tipo de adesivo, com quatro níveis, e da razão de compactação, com quatro níveis. Realizaram-se oito repetições para cada situação.

Tabela 2. Classificação da perda de massa em ensaio de resistência a fungos xilófagos.

Table 2. Mass loss classification in decay resistance test.

\begin{tabular}{ccc}
\hline Perda de Massa (\%) & Massa Residual (\%) & Classe de Resistência \\
\hline $0-10$ & $90-100$ & Altamente resistente \\
$11-24$ & $76-89$ & Resistente \\
$25-44$ & $56-75$ & Moderadamente resistente \\
$\geq 45$ & $\leq 55$ & Não resistente \\
\hline
\end{tabular}

Fonte: ASTM D - 2017 (2005b). 
Os dados, em porcentagem de perda de massa, foram transformados em arcsen [raiz quadrada (valor em porcentagem/100)], como sugerido por Steel \& Torrie (1980). Estas transformações foram necessárias em função da necessidade de normalizar a distribuição dos dados (teste de Lilliefors) e homogeneizar as variâncias (teste de Cochran e Bartellet). Para os fatores e a interação detectados como significativos pelo teste de $\mathrm{F}$, foi empregado o teste de Tukey, a 5\% de probabilidade.

\section{RESULTADOS E DISCUSSÃO}

\subsection{Resistência a fungos apodrecedores}

Na Tabela 3, constam os valores médios referentes à perda de massa (\%) dos painéis em função da razão de compactação e do tipo de adesivo, com as respectivas classes de resistência (Tabela 2).
De acordo com a ASTM D - 2017 (2005b), os painéis foram classificados como de moderada resistência ao fungo Gloeophyllum trabeum, com exceção do tratamento em que se empregaram $70 \%$ de ureia-formaldeído e $30 \%$ de taninos, e razão de compactação de 2,00, que foi classificado como resistente. Este tratamento proporcionou um ganho de resistência de 66,96\%, quando comparado àquele, para o mesmo adesivo, em que se empregou uma razão de compactação de 1,39. Como a composição adesívica foi a mesma, provavelmente o fungo tenha encontrado mais dificuldade em translocar suas enzimas no painel mais compactado.

Ao comparar a resistência do painel em que se empregou 30\% de taninos (adesivo 4) com aquele produzido com a mesma razão de compactação $(2,00)$, porém sem a adição de taninos à resina ureia-formaldeído (adesivo 1), obteve-se um ganho de resistência de 63,25\%. Desta forma, a adição de
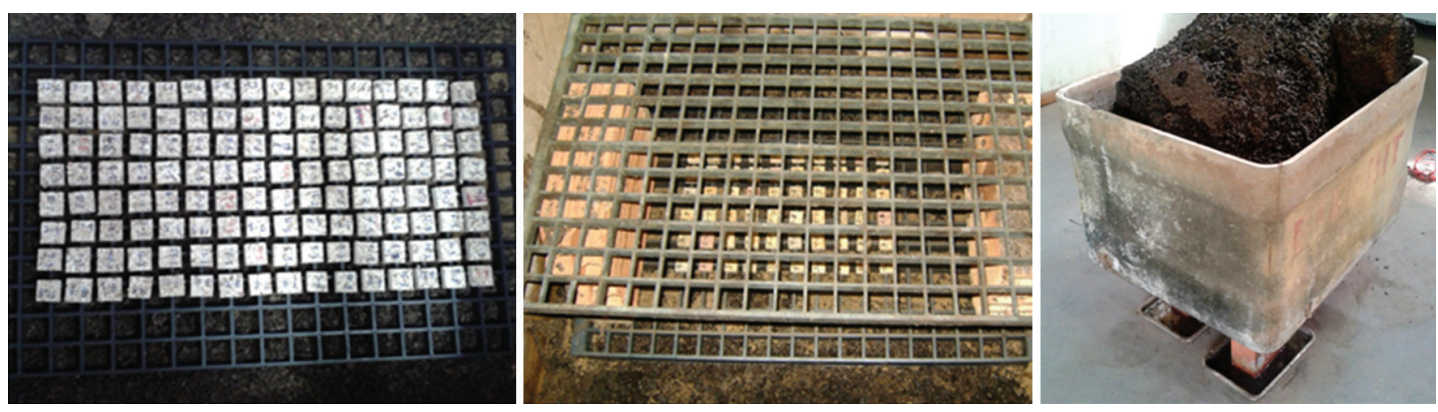

Figura 1. Montagem do ensaio de preferência alimentar com o térmita subterrâneo.

Figure 1. Mounting the feeding preference test with the subterranean termite.

Tabela 3. Perda de massa média causada pelos fungos xilófagos.

Table 3. Average mass loss caused by decay fungi.

\begin{tabular}{|c|c|c|c|c|}
\hline \multirow{3}{*}{$\begin{array}{c}\text { Razão de } \\
\text { Compactação }\end{array}$} & \multicolumn{4}{|c|}{ Perda de Massa (\%) para o Gloeophyllum trabeum } \\
\hline & \multicolumn{4}{|c|}{ Adesivos } \\
\hline & 1 & 2 & 3 & 4 \\
\hline 1,39 & $35,86 \mathrm{MR}$ & $41,17 \mathrm{MR}$ & $38,87 \mathrm{MR}$ & $38,05 \mathrm{MR}$ \\
\hline 1,55 & $36,17 \mathrm{MR}$ & $40,49 \mathrm{MR}$ & $36,80 \mathrm{MR}$ & $27,79 \mathrm{MR}$ \\
\hline 1,73 & $37,62 \mathrm{MR}$ & $40,57 \mathrm{MR}$ & $41,70 \mathrm{MR}$ & $39,44 \mathrm{MR}$ \\
\hline 2,00 & $34,20 \mathrm{MR}$ & $30,13 \mathrm{MR}$ & $37,77 \mathrm{MR}$ & $12,57 \mathrm{R}$ \\
\hline \multirow{3}{*}{$\begin{array}{c}\text { Razão de } \\
\text { Compactação }\end{array}$} & \multicolumn{4}{|c|}{ Perda de massa (\%) para o Trametes versicolor } \\
\hline & \multicolumn{4}{|c|}{ Adesivos } \\
\hline & 1 & 2 & 3 & 4 \\
\hline 1,39 & $24,01 \mathrm{R}$ & $19,96 \mathrm{R}$ & $22,03 \mathrm{R}$ & $21,19 \mathrm{R}$ \\
\hline 1,55 & $19,12 \mathrm{R}$ & $17,72 \mathrm{R}$ & $21,64 \mathrm{R}$ & $20,20 \mathrm{R}$ \\
\hline 1,73 & $17,67 \mathrm{R}$ & $20,12 \mathrm{R}$ & $21,13 \mathrm{R}$ & $21,29 \mathrm{R}$ \\
\hline 2,00 & $16,59 \mathrm{R}$ & $24,52 \mathrm{R}$ & $19,35 \mathrm{R}$ & $19,00 \mathrm{R}$ \\
\hline
\end{tabular}

$\mathrm{R}=$ resistente; $\mathrm{MR}=$ moderada resistência, segundo a ASTM D 2017 (2005b). 
taninos proporcionou melhora na resistência do painel. De acordo com Vital et al. (2001) e Colli et al. (2007), substâncias tânicas atuam na proteção do vegetal contra o ataque de micro-organismos, proporcionando aumento na resistência da madeira.

Para o fungo Trametes versicolor, todos os tratamentos (adesivos e razões de compactação) foram classificados como resistentes. De modo geral, observou-se uma tendência de melhoria da resistência dos painéis com o aumento da razão de compactação, porém sem nenhuma relação com o aumento da proporção de taninos à resina ureiaformaldeído.

Os valores foram analisados estatisticamente e houve diferença para os parâmetros adesivos, razões de compactação e fungos, e para as interações 'adesivos $\times$ fungos' e 'fungos $\times$ razões de compactação'. As interações foram desdobradas e analisadas pelo teste de Tukey a $5 \%$ de probabilidade (Tabelas 4 ).

Na Tabela 4, observa-se que o fungo Gloeophyllum trabeum causou perdas de massa maiores do que o Trametes versicolor para todos os adesivos e razão de compactação testados. Entre os adesivos, o 4 (70\% ureia-formaldeído e 30\% taninos) foi o que promoveu a menor perda de massa para o fungo Gloeophyllum trabeum. Não houve diferença entres os adesivos testados para o fungo Trametes versicolor. Melo et al. (2010), ao estudarem a resistência de painéis aglomerados com seis diferentes proporções de madeira (Eucalyptus grandis) e casca de arroz colados com adesivos à base de ureia-formaldeído e tanino-formaldeído, não encontraram diferença significativa entre as perdas de massa causadas pelo Trametes versicolor para os adesivos testados.
Ujang \& Amburgey (1993), ao estudarem a resistência natural da madeira proveniente de árvores de Acacia mangium com cinco anos de idade aos fungos Gloeophyllum trabeum e Trametes versicolor, encontraram que o Gloeophyllum trabeum causou perda de massa de $34,40 \%$, enquanto a perda provocada pelo Trametes versicolor foi de 27,59\%.

Para o fungo Gloeophyllum trabeum, a razão de compactação 2,00 proporcionou maior resistência aos painéis quando comparada às razões de compactação de 1,39 e 1,73, enquanto a de 1,55 proporcionou resistência intermediária entre a razão de 2,00 e as demais. Já para o Trametes versicolor, não houve diferença entre as perdas de massa para as razões de compactação testadas.

\subsection{Resistência a térmitas xilófagos em ensaio de preferência alimentar}

Depois de decorridos 45 dias, quando da desativação do ensaio, observou-se que os térmitas haviam consumido por completo as amostras, independentemente do adesivo ou da razão de compactação empregada.

Isto ocorreu em consequência do número de indivíduos existentes na colônia utilizada e da baixa resistência da madeira de Acacia mangium ao térmita empregado. Guerra (2010), ao testar cinco madeiras ao térmita Nasutitermes sp. testado neste trabalho, detectou que a madeira de Acacia mangium, mesmo não sendo totalmente consumida, foi aquela que apresentou uma menor resistência, atingindo perda de massa de até $49 \%$.

Tabela 4. Comparação entre médias das perdas de massa para fungos, adesivos e razões de compactação testados. Table 4. Comparison among averages of mass loss to decay fungi, adhesives and compaction ratios tested.

\begin{tabular}{|ccccc|}
\multirow{2}{*}{ Fungos } & \multicolumn{4}{c|}{ Adesivos } \\
\cline { 2 - 5 } & $\mathbf{1}$ & $\mathbf{2}$ & $\mathbf{3}$ & $\mathbf{4}$ \\
Gloeophyllum trabeum & $35,96 \mathrm{Aa}$ & $38,09 \mathrm{Aa}$ & $38,78 \mathrm{Aa}$ & $29,46 \mathrm{Ba}$ \\
Trametes versicolor & $19,35 \mathrm{Ab}$ & $20,57 \mathrm{Ab}$ & $21,04 \mathrm{Ab}$ & $20,41 \mathrm{Ab}$ \\
\hline \multicolumn{1}{c}{ Fungos } & $\mathbf{1 , 3 9}$ & $\mathbf{R a z} \tilde{e}$ es de compactação \\
\cline { 2 - 5 } & $38,48 \mathrm{Aa}$ & $\mathbf{1 , 5 5}$ & $\mathbf{1 , 7 3}$ & $\mathbf{2 , 0 0}$ \\
Gloeophyllum trabeum & $21,79 \mathrm{Ab}$ & $19,31 \mathrm{ABa}$ & $39,83 \mathrm{Aa}$ & $28,66 \mathrm{Ba}$ \\
Trametes versicolor & & $20,05 \mathrm{Ab}$ & $19,86 \mathrm{Ab}$ \\
\hline
\end{tabular}

As médias seguidas da mesma letra, maiúscula na horizontal ou minúscula na vertical, não diferem estatisticamente pelo teste de Tukey $(\mathrm{p} \geq 0,05)$. 


\section{CONCLUSÕES}

De modo geral, a maior proporção de taninos (adesivo $4-30 \%$ de taninos) e a maior razão de compactação testada $(2,00)$ promoveram maior resistência aos painéis em relação ao ataque do fungo Gloeophyllum trabeum.

As razões de compactação e as proporções de taninos adicionadas à ureia-formaldeído não tiveram efeito quanto ao ataque do fungo Trametes versicolor.

O fungo Gloeophyllum trabeum causou maior perda de massa aos painéis testados quando comparado com o Trametes versicolor.

Os painéis produzidos com a madeira de Acacia mangium e colados com adesivo à base de ureia-formaldeído e taninos, para as razões de compactação testadas, não exibiram resistência ao ataque e foram completamente consumidos pelos térmitas do gênero Nasutitermes sp.

\section{STATUS DA SUBMISSÃO}

Recebido: 11 set., 2013

Aceito: 8 maio, 2014

Publicado: 28 ago., 2014

\section{AUTOR(ES) PARA CORRESPONDÊNCIA}

Juarez Benigno Paes

Departamento de Ciências Florestais e da

Madeira, Universidade Federal do Espírito

Santo - UFES, CEP 29550-000, Jerônimo

Monteiro, ES, Brasil

e-mail: jbp2@uol.com.br

\section{REFERENNCIAS}

American Society for Testing and Materials - ASTM. ASTM D-1413: Standard test method for wood preservatives by laboratory soil-block cultures. Philadelphia; 2005a. 7 p. Annual Book of ASTM Standard.

American Society for Testing and Materials - ASTM. ASTM D-2017: Standard method of accelerated laboratory test of natural decay resistance of woods. Philadelphia; 2005b. 5 p. Annual Book of ASTM Standard.

American Society for Testing and Materials - ASTM. ASTM D-3345: Standard method for laboratory evaluation of the wood and other cellulosic materials for resistance to termite. Philadelphia; 2005c. 3 p. Annual Book of ASTM Standard.

Colli A, Nascimento AM, Xavier LM, Rubim IB. Propriedades físico-mecânicas e preservação, com boro e tanino do Bambusa tuldoides (Munro). Floresta e Ambiente 2007; 14(1): 56-64.

Dacosta LPE. Utilização de resíduos do processamento mecânico da madeira para a fabricação de chapas aglomeradas [dissertação]. Santa Maria: Universidade Federal de Santa Maria; 2004.

Dunky M, Pizzi A. Wood adhesives. In: Chaudhury M, Pocius AV, editors. Adhesive science and engineering. Amsterdam: Elsevier; 2002.

Gonçalves FG. Painéis aglomerados de madeira de Acacia mangium com adesivos de uréia formaldeído e tanino em pó da casca de Acacia mearnsii [tese]. Seropédica: Universidade Federal Rural do Rio de Janeiro; 2012.

Guerra SCS. Influência do teor de extrativos na resistência natural de cinco madeiras a cupins xilófagos [monografia]. Jerônimo Monteiro: Universidade Federal do Espírito Santo; 2010.

Iwakiri S. Painéis de madeira reconstituída. Curitiba: FUPEF; 2005.

Lima AM, Vidaurre GB, Lima RM, Brito EO. Utilização de fibras (epicarpo) de babaçu como matéria-prima alternativa na produção de chapas de madeira aglomerada. Revista Árvore 2006; 30(4): 645-650. http:// dx.doi.org/10.1590/S0100-67622006000400018

Maloney TM. Modern particleboard and dry-process fibreboard manufacturing. 2. ed. São Francisco: Miller Freeman; 1993.

Melo RR. Propriedades físico-mecânicas e resistência a biodeterioradores de chapas aglomeradas constituídas por diferentes proporções de madeira e casca de arroz [dissertação]. Santa Maria: Universidade Federal de Santa Maria; 2009.

Melo RR, Santini EJ, Haselein CR, Garlet A, Paes JB, Stangerlin DM. Resistência de painéis aglomerados produzidos com diferentes proporções de madeira e casca de arroz a fungos e cupins xilófagos. Ciência Florestal 2010; 20(3): 501-511.

Moslemi AA. Particleboard. London: Southern Illinois University Press; 1974.

Ndazi B, Tesha JV, Karlsson S, Bisanda ETN. Production of rice husks composites with Acacia mimosa tannin-based resin. Journal of Materials Science 2006; 41(21): 6978-6983. http://dx.doi. org/10.1007/s10853-006-0220-7

Paes JB, Ramos IEC, Farias Sobrinho DW. Eficiência do CCB na resistência da madeira de algaroba (Prosopis juliflora (Sw) D.C.) a cupins subterrâneos (Nasutiternes 
corniger motsch.) em ensaio de preferência alimentar. Ambiência 2006; 2(1): 51-64.

Paes JB, Melo RR, Lima CR, Oliveira O. Resistência natural de sete madeiras ao cupim subterrâneo (Nasutitermes corniger Motsch.) em ensaio de preferência alimentar. Revista Brasileira de Ciências Agrárias 2007; 2(1): 57-62.

Pauleski DT, Haselein CR, Santini EJ, Rizzatti E. Características de compósitos laminados manufaturados com polietileno de alta densidade (PEAD) e diferentes proporções de casca de arroz e partículas de madeira. Ciência Florestal 2007; 17(2): 157-170.

Steel RGD, Torrie JH. Principles and procedures of statistic: a biometrical approach. 2nd ed. New York: McGraw Hill; 1980.

Tostes AS, Lelis RCC, Pereira KRM, Brito EO. Colagem de chapas de madeira aglomerada com adesivo ureia-formaldeído (UF) modificado com tanino da casca de Eucalyptus pellita F. Muell. Floresta e Ambiente 2004; 11(1): 15-19.

Trianoski R. Avaliação do potencial de espécies florestais alternativas, de rápido crescimento, para produção de painéis de madeira aglomerada [dissertação]. Curitiba: Universidade Federal do Paraná; 2010.

Vieira MC, Brito EO, Gonçalves FG. Evolução econômica do painel compensado no Brasil e no mundo. Floresta e Ambiente 2012; 19 (3): 277-285. http://dx.doi. org/10.4322/floram.2012.033

Vital BR, Shimada AN, Valente OF, Della Lucia RM, Pimenta AS. Avaliação dos taninos da casca de Eucalyptus grandis W. Hill ex Maiden como preservativo de madeira. Revista Árvore 2001; 25(2): 245-256.

Ujang S, Amburgey TL. Decay resistance of Acacia mangium heartwood against brown-and white-rot fungi: preliminary results. Journal of Tropical Forest Science 1993; 6(1): 16-20. 